\title{
Resection of "down-staged" advanced hepatocellular carcinoma after treatment with the VEGFR2 inhibitor apatinib: five cases report
}

\author{
Zhenyu Hou, Keyun Zhu, Xuejiao Yang, Hongyuan Zhou, Ping Chen, Ge Yu, Xiaolin Zhu, Yunlong Cui, \\ Tianqiang Song, Qiang Li, Huikai Li, Ti Zhang \\ Department of Hepatobiliary Surgery, Tianjin Medical University Cancer Institute and Hospital, Key Laboratory of Cancer Prevention and \\ Therapy, National Clinical Research Center for Cancer, Tianjin, China \\ Correspondence to: Ti Zhang, MD, PhD; Huikai Li, MD. Department of Hepatobiliary Surgery, Tianjin Medical University Cancer Institute and \\ Hospital, Key Laboratory of Cancer Prevention and Therapy, National Clinical Research Center for Cancer, 24 Bin Shui Road, Hexi District, \\ Tianjin 300060, China. Email: zhangti@tjmuch.com; tjchlhk@126.com.
}

\begin{abstract}
Sorafenib and lenvatinib are currently standard treatments for advanced hepatocellular carcinoma (HCC); however, the therapeutic effect is unsatisfying. Indeed, very few patients with HCC under sorafenib treatment were eligible for surgery in the past ten years. In addition, there is no report of a patient with the opportunity to undergo radical resection after treatment with lenvatinib. Here, we describe five patients with advanced and unresectable HCC that were able to receive curative resection within 1 year of treatment with the tyrosine kinase inhibitor apatinib that selectively inhibits vascular endothelial growth factor receptor 2 (VEGFR2). The five patients with advanced and unresectable HCC were treated with apatinib (250 mg po, qd), and all the five patients obtained an objective response to the treatment, allowing for subsequent resection, and the second patient even obtained a pathological complete response. The latest follow-up date was August 20, 2019, and all patients were alive at the latest follow-up. The disease-free survival of the first patient was 13 months. Lung metastasis was found 12 months later after surgery for patient 5 . The other three patients have no recurrence. This is the first report of a single drug with promising therapeutic effects in patients with advanced HCC within one year at a single center. Therefore, apatinib may be promising for some patients with locally advanced HCC to undergo radical resection and improve outcomes.
\end{abstract}

Keywords: Apatinib; hepatocellular carcinoma (HCC); resection; vascular endothelial growth factor receptor 2 (VEGFR2); case report

Submitted Dec 31, 2019. Accepted for publication Jul 03, 2020.

doi: $10.21037 /$ tcr-19-3019

View this article at: http://dx.doi.org/10.21037/tcr-19-3019

\section{Introduction}

Hepatocellular carcinoma (HCC) is the fifth most common type of cancer and the third highest cause of cancer-related mortality worldwide (1). Portal vein tumor thrombosis (PVTT) is identified in $10-40 \%$ of patients with HCC at the time of initial diagnosis (2). According to the Barcelona Clinic Liver Cancer (BCLC) staging system (3), sorafenib and lenvatinib are recommended first-line treatment for advanced HCC. In addition, regorafenib is recommended in second-line in those patients who progressed but were tolerant to sorafenib. Cabozantinib and nivolumab also show survival benefit in second-line treatment. However, in the SHARP and ORIENTAL clinical trials, the partial response (PR) to sorafenib was only $2 \%$ and $3.3 \%$, and the overall survival (OS) was only 10.7 and 6.8 months, respectively $(4,5)$. Although the median OS of lenvatinib was found to be slightly higher at 13.6 months in the REFLECT study, its efficacy was not inferior to that of sorafenib (6).

In the past decade, many attempts have been made to 
treat advanced HCC, such as systematic chemotherapy, internal radiation with Y-90 resin microspheres and targeted drugs (7-12). However, none of them have been shown to be more effective than sorafenib. Although not recommended by the guidelines, some other treatments are widely used for advanced HCC, such as hepatectomy, transarterial chemoembolization (TACE), hepatic arterial infusion chemotherapy, and external beam radiation therapy (13). Hepatectomy is a very effective treatment but is only available for some highly selected patients. Moreover, the prognosis of these patients after surgery varies according to the extent of tumor thrombosis, in which patients with $\mathrm{Vp} 1$ PVTT may achieve a great prognosis (14-17). Therefore, more effective treatment modalities are needed for advanced HCC patients, especially for locally advanced cases.

The success of immune checkpoint inhibitors in different tumors has stimulated several clinical trials of different checkpoint inhibitors in HCC. Nivolumab, a programmed cell death protein-1 (PD-1) immune checkpoint inhibitor has been approved by the Food and Drug Administration for treating advanced HCC (18). Several preclinical studies have reported promising antitumor effects in combination with anti-angiogenic drugs and immunotherapy in a variety of tumor types (19). Therefore, this combination therapy has received considerable attention. Recent studies have suggested that PD-1 antibody combined with antiangiogenesis therapy may be a promising method for the treatment of HCC and the ORR can achieved 40-50\% $(20,21)$. ESMO 2018 reported that the ORR can achieved $34 \%$ when combined PD-L1 monoclonal antibody with bevacizumab. And ASCO GI 2019 reported a $37.5 \%$ pathological complete response when combined CTLA-4 antibody with PD-1 monoclonal antibody for neoadjuvant therapy. These studies provide new hope for advanced HCC (22).

It is well accepted that some patients with initially unresectable advanced colorectal cancer have a highly increased opportunity to receive curative treatment after neoadjuvant chemotherapy (23-25). By contrast, very few patients with advanced HCC show a sufficient response to allow for curative resection after treatment with sorafenib (26-29). Recently, we reported that apatinib, an orally administered small-molecule inhibitor of vascular endothelial growth factor 2 (VEGFR2) demonstrated novel therapeutic effects for patients with advanced HCC (30). Twenty-two patients were enrolled in the study, the PR rate was $40.9 \%$ and the median time to progression of treated patients was even achieved 10.4 months. Here, we report five additional patients with locally advanced HCC that also showed a good response to apatinib, leading to significant cytoreduction that enabled curative resection within 1 year. We present the following article in accordance with the CARE reporting checklist (available at http://dx.doi. org/10.21037/tcr-19-3019).

\section{Case presentation}

All procedures performed in this study were in accordance with the ethical standards of the institutional committee and with the Helsinki Declaration (as revised in 2013). Written informed consent was obtained from the patient.

\section{Case one}

Case one involves a male patient of 46 years old, who was diagnosed with advanced HCC on July 25, 2016 at Tianjin Medical University Cancer Institute and Hospital. The patient was suffering from chronic hepatitis B virus (HBV) infection, and the HBV-DNA titer was $3.63 \times 10^{6} / \mathrm{L}$. Enhanced MRI scans revealed a mass about $10.3 \mathrm{~cm} \times$ $7.6 \mathrm{~cm}$ in the left lobe of the liver with typical HCC radiological characteristics, and tumor thrombosis in the left portal vein and left hepatic vein. The serum level of alpha fetoprotein (AFP) was $28,295 \mathrm{ng} / \mathrm{mL}$. The patient had liver cirrhosis and poor liver function. The serum total bilirubin level was $27.1 \mu \mathrm{mol} / \mathrm{L}$ and the indocyanine green (ICG)-R15 was $18.4 \%$. Considering the large tumor size with vessel involvement, severe liver cirrhosis, as well as high virus load, the patient was considered to be at high risk for postoperative liver failure. Therefore, we first carried out antiviral and liver preservation treatments, and then performed TACE treatment twice. Unfortunately, MRI examination on October 11, 2016 showed that the size of the tumor mass increased to $10.6 \mathrm{~cm} \times 8.5 \mathrm{~cm}$, and a new lesion was detected in the left lobe. Meanwhile, the serum AFP level further increased to $36,671 \mathrm{ng} / \mathrm{mL}$. The treatment response to TACE was classified as progressive disease according to the RECIST criteria. Therefore, we ceased TACE treatment and suggested treatment with apatinib (250 mg, po, qd) based on our previous experience (30).

The patient was then under routine follow up. On November 22, 2016, MRI revealed that the tumor size reduced to $9.1 \mathrm{~cm} \times 7.6 \mathrm{~cm}$, and the serum AFP level decreased to $33,829 \mathrm{ng} / \mathrm{mL}$. Subsequently, the tumor diameter and AFP level gradually decreased, reaching $6.5 \mathrm{~cm}$ and $3,473 \mathrm{ng} / \mathrm{mL}$, respectively, after 6 months of 
A
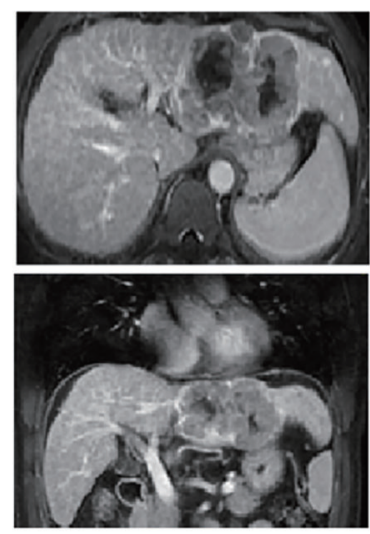

2016.06.27

Before treatment
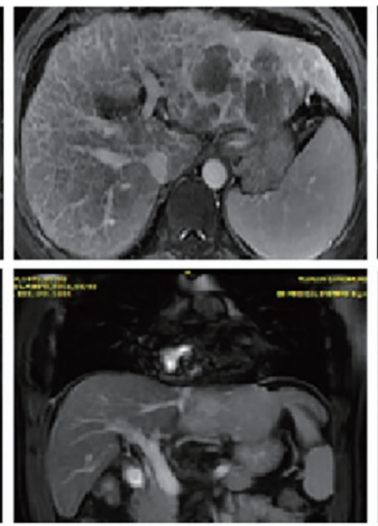

2016.10.11 After TACE
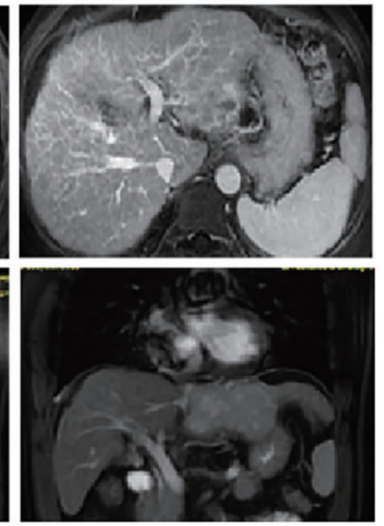

2017.04.12 After apatinib
B
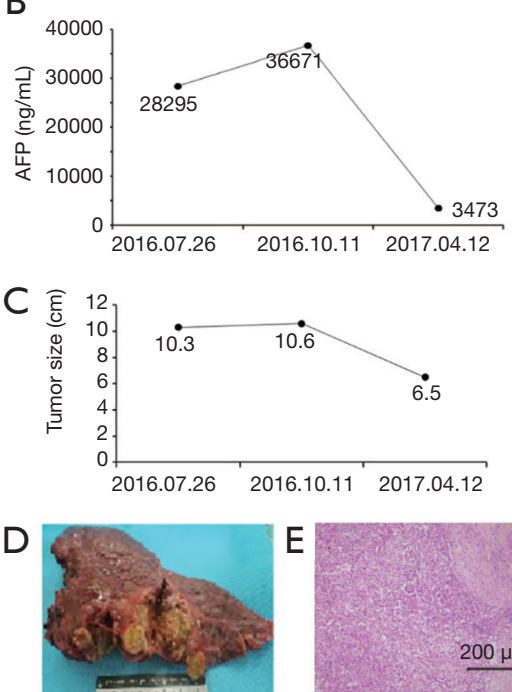

Figure 1 Clinical data of Case 1. (A) Change of tumor size assessed by enhanced magnetic resonance imaging (MRI). (B) Clinical course of serumalpha fetoprotein (AFP) levels. (C) Clinical course of the tumor size. (D) Gross findings of the resected specimen. Three lesions encapsulated by fibrotic tissue were detected. (E) histopathological finding of the resected specimen; the tumor was moderately differentiated HCC, and massive necrosis was observed. TACE, transarterial chemoembolization; HCC, hepatocellular carcinoma.

apatinib treatment. Importantly, the vessel involvement was also significantly relieved, and liver function improved: total bilirubin was $20.6 \mu \mathrm{mol} / \mathrm{L}$ and ICG-R15 was $10.4 \%$. Considering the dramatically reduced tumor size and improved liver function, this patient was suitable for surgical treatment. We first ceased the use of apatinib for 13 days, and then preformed a left lateral lobe hepatectomy on April 26, 2017. Postoperative pathology revealed moderately differentiated HCC with tumor thrombosis in the left portal vein and the left hepatic vein. Massive necrosis was observed in the tumor (Figure 1). Seventeen days after the operation, the patient continued to take apatinib. The serum AFP dropped to a normal level within two months after the surgery.

\section{Case two}

Case two involves a male of 64 years old, who was diagnosed with advanced HCC on July 26, 2017. Enhanced MRI scans revealed a mass of approximately $12.8 \mathrm{~cm} \times 9.4 \mathrm{~cm}$ in the left lobe of the liver, and tumor thrombosis in the main portal vein, left portal vein, middle hepatic vein, left hepatic vein, and the inferior vena cava (IVC). The serum AFP level was $28,765 \mathrm{ng} / \mathrm{mL}$. Given the PVTT in the main portal vein and tumor thrombosis in the IVC, we recommended treatment of apatinib (250 mg, po, qd) directly. Because the patient had chronic hepatitis $\mathrm{C}$ virus infection, and the HCV-RNA titer was $2.60 \times 10^{6} / \mathrm{L}$, sofosbuvir and daclatasvir were also used for antiviral therapy.

At each follow-up visit, the tumor diameter and AFP level decreased synchronously. On October 26, 2017, MRI revealed that the tumor size reduced to $6.2 \mathrm{~cm} \times 3.2 \mathrm{~cm}$ and the AFP level decreased to $54.82 \mathrm{ng} / \mathrm{mL}$. The treatment response was classified as PR according to the RECIST criteria. We continued to treat the patient with apatinib, and the tumor and tumor thrombosis were further reduced. Meanwhile, the AFP level decreased to $4.62 \mathrm{ng} / \mathrm{mL}$ and remained stable below $5 \mathrm{ng} / \mathrm{mL}$ for the next 3 months. On April 2, 2018, MRI revealed the lesion size of $4.4 \mathrm{~cm} \times 3.2 \mathrm{~cm}$ and the AFP level was $3.06 \mathrm{ng} / \mathrm{mL}$. Seventeen days after ceasing apatinib, we performed a left hemi-hepatectomy together with tumor thrombosis in the IVC. Postoperative pathology revealed necrosis in the entire tumor and tumor thrombosis, and no live tumor cells were found. Therefore, this patient had a pathological complete response (Figure 2).

\section{Case three}

Case three involves a 67-year-old male, who was diagnosed with advanced HCC on October 28, 2017. Enhanced MRI 
A

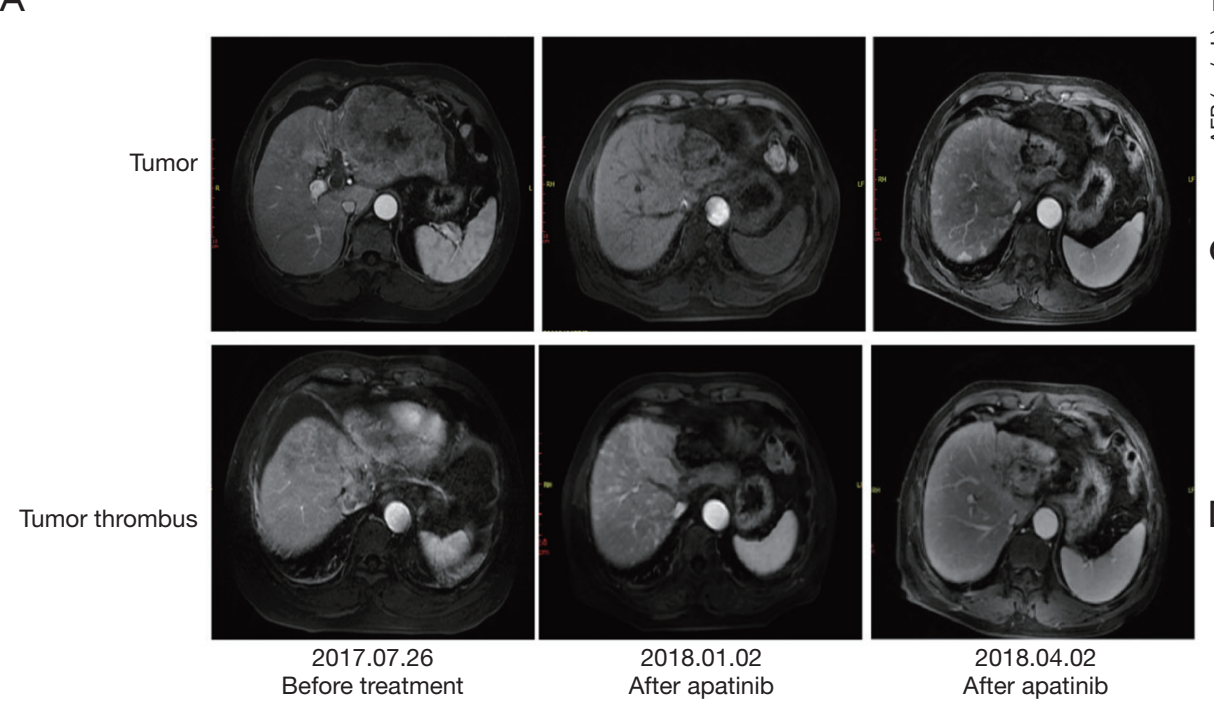

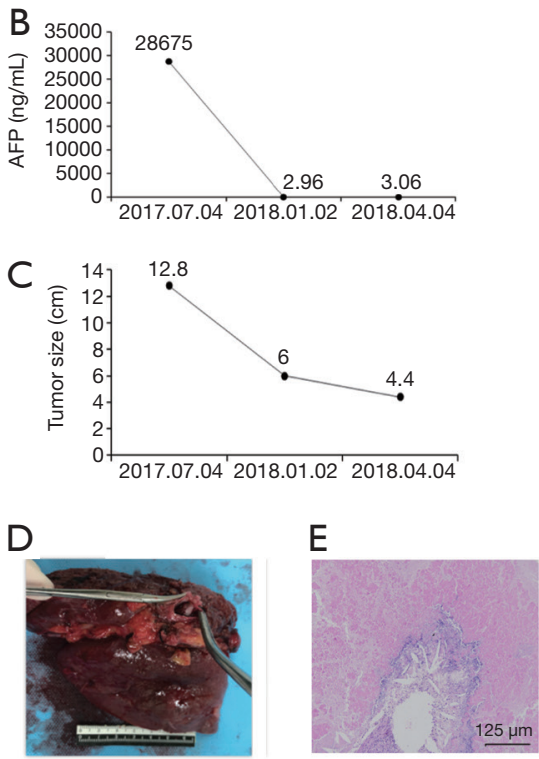

Figure 2 Clinical data of Case 2. (A) Change of tumor size and tumor thrombus assessed by enhanced magnetic resonance imaging (MRI); (B) clinical course of serum alpha fetoprotein (AFP) levels and tumor size; (C) clinical course of tumor size; (D) gross findings of the resected specimen; (E) histopathological finding of the resected specimen; only necrotic tissues were observed, and no tumor cells were found.

scans revealed a mass of about $2.8 \mathrm{~cm} \times 1.9 \mathrm{~cm}$ in the hepatic hilar region, with tumor thrombus in the left hepatic vein, IVC, common bile duct, and left hepatic bile duct. The serum AFP level was $1,714 \mathrm{ng} / \mathrm{mL}$ and the total bilirubin level was $303.1 \mu \mathrm{mol} / \mathrm{L}$. The patient had chronic hepatitis $\mathrm{B}$, and his HBV DNA viral titer was $1.17 \times 10^{6} / \mathrm{L}$. Entecavir was used as antiviral treatment. Given the tumor thrombosis in the choledochus and high total bilirubin level, the patient was also diagnosed with obstructive jaundice. The patient first received percutaneous transhepatic cholangial drainage. One month later, the total bilirubin level decreased to $38.4 \mu \mathrm{mol} / \mathrm{L}$, and the patient was started on apatinib (250 mg, po, qd). The serum AFP declined progressively at each follow-up visit. On February 6, 2018, MRI revealed reduction in the lesion size along with necrosis and shrinkage of tumor thrombosis in the left portal vein, IVC, and left hepatic bile duct. After 1 month of stopping the use of apatinib, the patient received an expanded left hemihepatectomy, total caudate lobectomy, cholecystectomy, right hepatic choledochojejunostomy, and jejunal end-to-side anastomosis (Roux-en-Y). Postoperative pathology revealed moderately differentiated HCC with tumor thrombosis in the left hepatic vein and the left hepatic bile duct. Massive necrosis was also observed in the tumor (Figure 3).

Case four
Case 4 involves a 46-year-old male, who was diagnosed with advanced HCC on August 1,2017. Enhanced MRI scans revealed a mass of approximately $16.4 \mathrm{~cm} \times 16.3 \mathrm{~cm}$ in the right lobe of the liver with typical HCC radiological characteristics, and tumor thrombus in the right portal vein. The AFP level was $1,032 \mathrm{ng} / \mathrm{mL}$. Therefore, we suggested a treatment course of apatinib $(250 \mathrm{mg}$, po, qd) and performed TACE treatment on August 3, 2017, September 3, 2017, October 3, 2017, December 22, 2017, and March 1, 2018. Enhanced MRI was performed before each TACE treatment, revealing a tumor size of $16.4 \mathrm{~cm} \times 16.3 \mathrm{~cm}$, $16.3 \mathrm{~cm} \times 15.5 \mathrm{~cm}, 16.3 \mathrm{~cm} \times 10.5 \mathrm{~cm}, 13.7 \mathrm{~cm} \times 13.5 \mathrm{~cm}$, and $13.1 \mathrm{~cm} \times 12.8 \mathrm{~cm}$, respectively. Fourteen days after ceasing apatinib (May 2, 2018), we preformed segmentectomy (VIII). Postoperative pathology revealed moderately differentiated HCC with massive necrosis and negative margins (Figure 4).

\section{Case five}

Case 5 involves a 68-year-old male, who was diagnosed with advanced HCC on October 20, 2017. Enhanced MRI scans revealed multiple masses in the right lobe of the liver with a maximum diameter of $4.8 \mathrm{~cm} \times 4.3 \mathrm{~cm}$. There was invasion of the right anterior branch of the portal vein and 
A
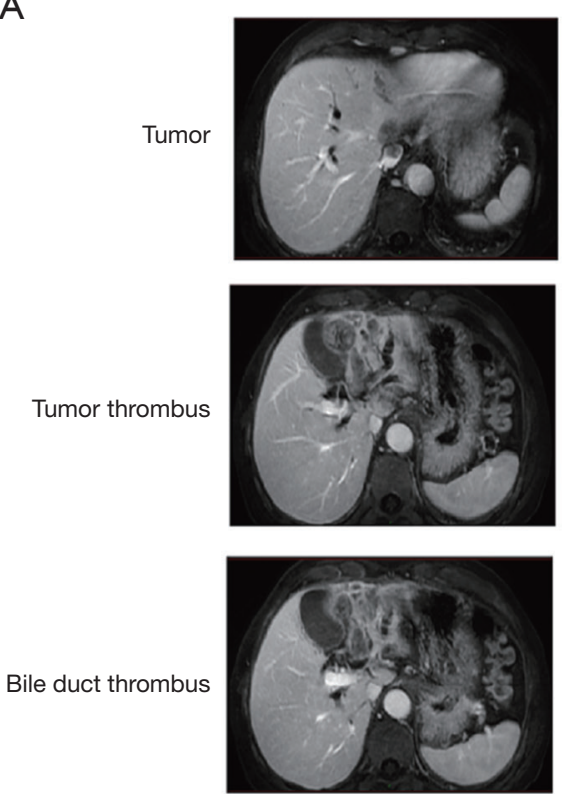

2017.10.08 Before treatment
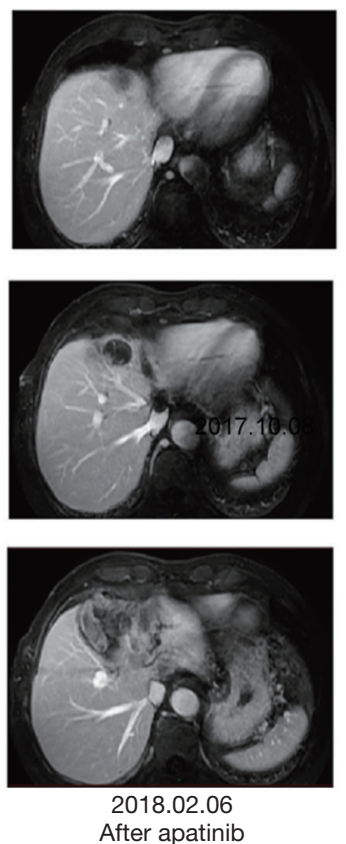

B

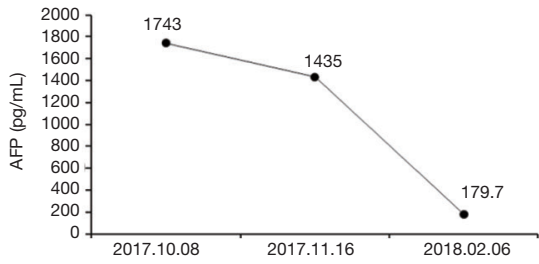

C
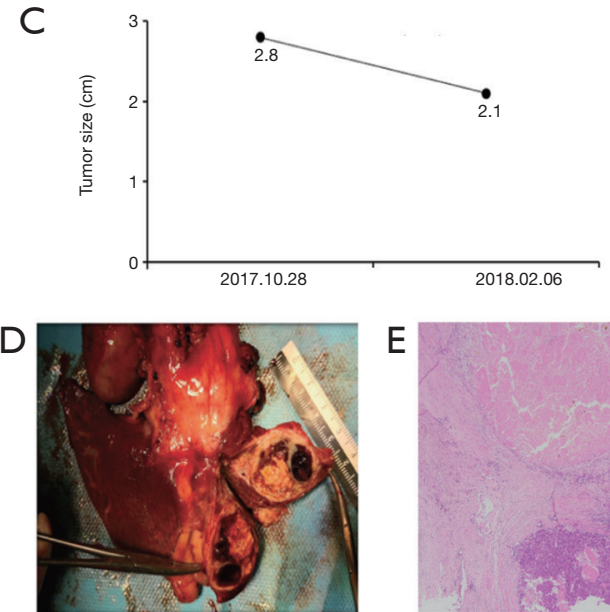

E

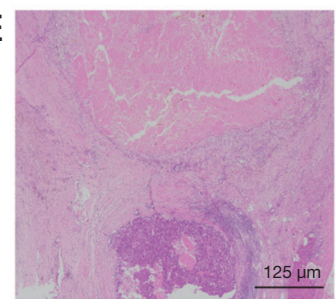

Figure 3 Clinical data of Case 3. (A) Change of tumor size and tumor thrombus assessed by enhanced magnetic resonance imaging (MRI); (B) clinical course of serum alpha fetoprotein (AFP) levels; (C) clinical course of tumor size; (D) gross findings of the resected specimen; most of the tumor showed necrosis; (E) histopathological finding of the resected specimen; necrotic tissues were observed, but some cells were still active.

second lumbar metastasis. The AFP level was $10.81 \mathrm{ng} / \mathrm{mL}$. The viral titer of HBV DNA was $2.15 \times 10^{4} / \mathrm{L}$, and entecavir was used as antiviral treatment. The patient had cirrhosis and the liver function was poor. TACE treatment was carried out three times, and apatinib was also administered (250 mg, po, qd) starting November 2, 2017. On April 4, 2018, enhanced MRI revealed that the lesion had shrunk, the right anterior branch PVTT disappeared, and the lesion on the second lumbar was also reduced. However, the AFP level increased to $32.31 \mathrm{ng} / \mathrm{mL}$. One month after ceasing apatinib (April 4, 2018), we performed liver segmentectomy (VI, VII), partial liver resection (V, VIII), and cholecystectomy. Postoperative pathology revealed poorly differentiated HCC with massive necrosis (Figure 5).

\section{Discussion}

Sorafenib is a small-molecule kinase inhibitor that inhibits the kinase activities of Raf-1 and B-Rafin addition to VEGFRs and platelet-derived growth factor receptor. However, the SHARP (4) and ORIENTAL (5) clinical trials showed that the median OS of patients in the sorafenib group was 10.7 and 6.5 months, with a PR rate of only $2 \%$ and $3.3 \%$, respectively. Recently, some other agents such as lenvatinib and regorafenib have been approved for use in the treatment of advanced HCC; however, their efficacy is not superior to that of sorafenib.

Surgical resection is generally believed to be the most effective therapeutic strategy for some highly selected HCC patients with PVTT in the first or higher-order branch of the portal vein. However, for patients with PVTT in the main trunk or with tumor thrombosis in the IVC, surgical resection cannot prolong survival (17). Accordingly, the majority of patients with locally advanced HCC have no chance to receive curative resection, and the prognosis is very poor even after sorafenib treatment.

Many patients with other cancer types, including colorectal cancer, that are also not suitable for surgery at the time of initial diagnosis could have the opportunity for surgery with an excellent prognosis after effective neoadjuvant chemotherapy (23-25). Although the success rate is lower than for colorectal cancer, cytoreduction or a 
A

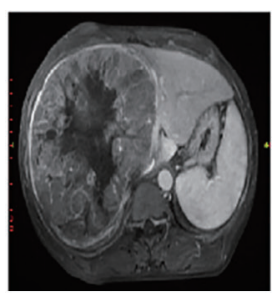

2017.07.28 Before treatment

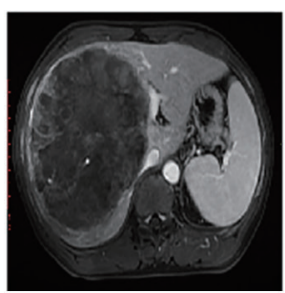

2017.12.12

After apatinib

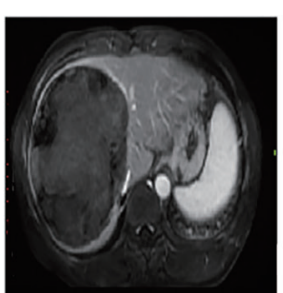

2018.04.16

After apatinib

\section{B}

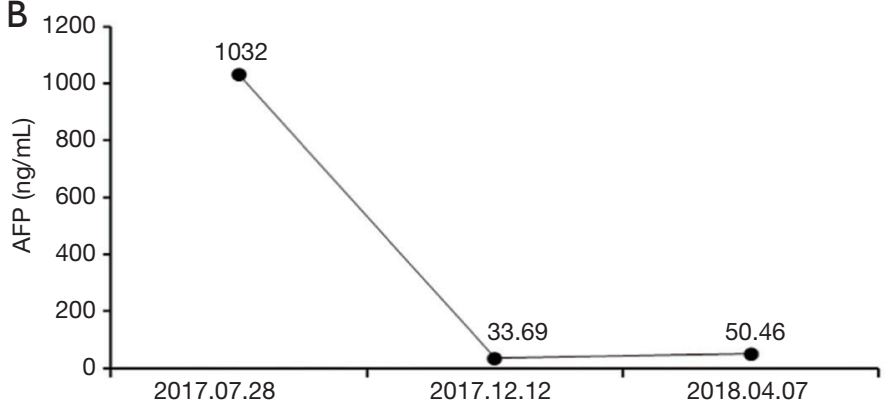

C

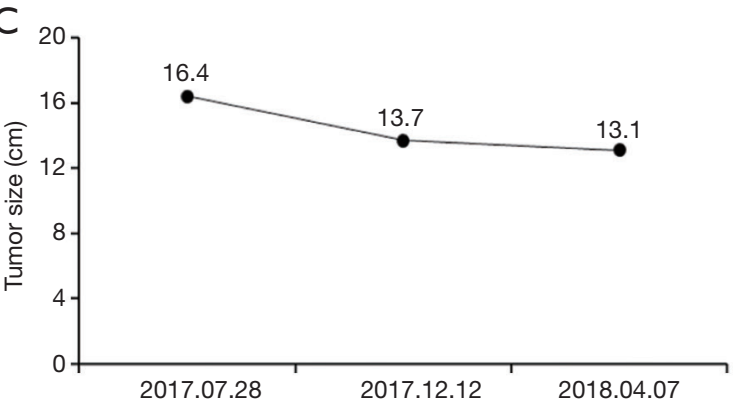

D

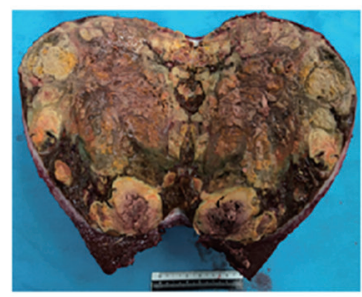

E

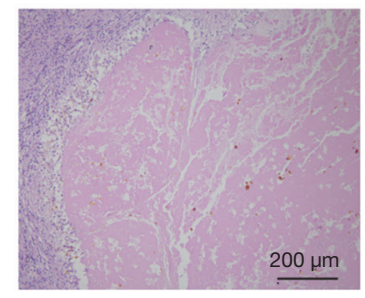

Figure 4 Clinical data of Case 4. (A) Change of tumor size and tumor thrombus assessed by enhanced magnetic resonance imaging (MRI); (B) clinical course of alpha fetoprotein (AFP) levels and tumor size; (C) clinical course of tumor size; (D) gross findings of the resected specimen; a large tumor was detected in the right lobe, and the majority of the tumor showed necrosis; (E) histopathological finding of the resected specimen; necrotic tissues were observed, but some cells were still active.

A

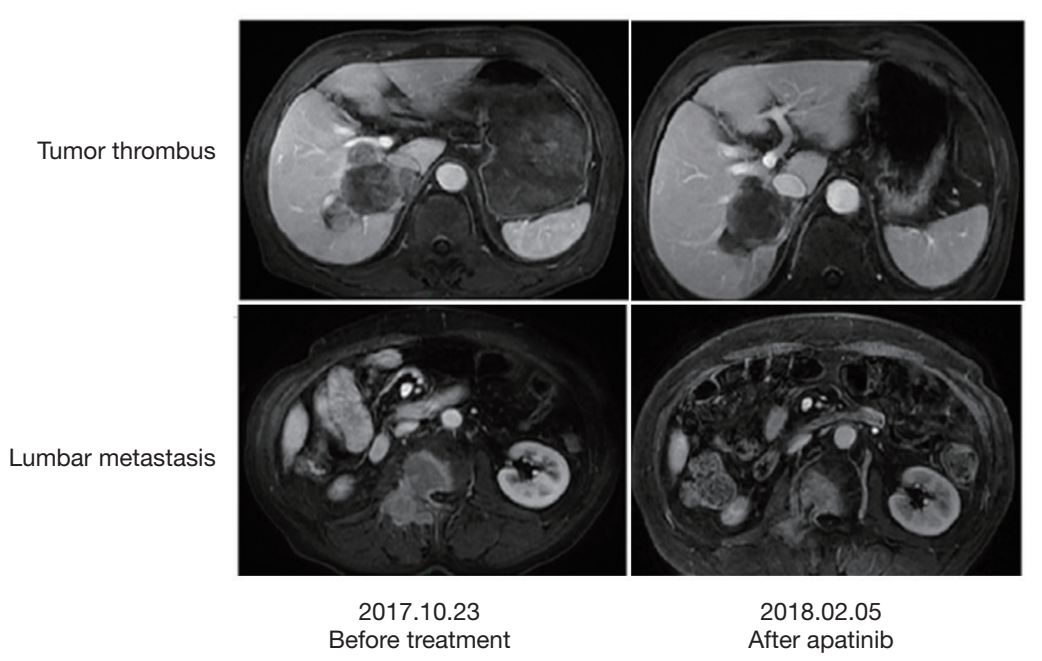

B

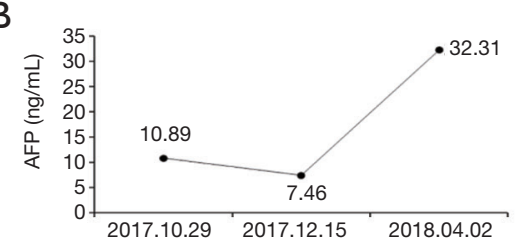

C
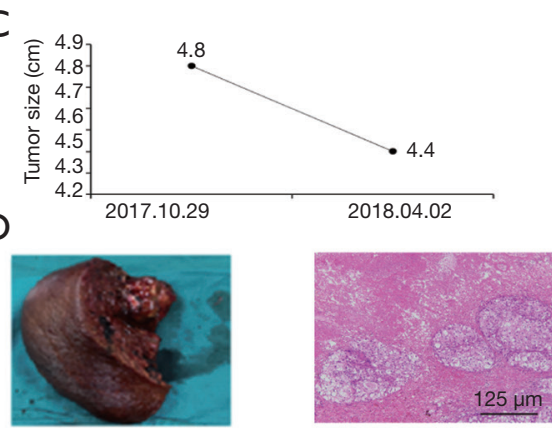

Figure 5 Clinical data of Case 5. (A) Change of tumor size and tumor thrombus assessed by enhanced magnetic resonance imaging (MRI); (B) clinical course of serum alpha fetoprotein (AFP) levels; (C) clinical course of tumor size; (D) gross findings of the resected specimen; the lesion in the right lobe was completely resected; (E) histopathological finding of the resected specimen. 
"down-staging" strategy for patients with locally advanced HCC may significantly improve the OS, or even provide the opportunity for resection (31). Indeed, some patients with advanced HCC achieved a PR or complete response, and even received subsequent surgical treatment after sorafenib treatment (26-29). However, such cases are still relatively rare on the global scale, and there has been no similar case reported after taking lenvatinib.

We previously reported that apatinib had an unprecedented therapeutic effect for patients with advanced HCC, in which $40.9 \%$ of the patients achieved a PR (30). In our phase II clinical trial (NCT03046979), apatinib showed robust clinical activity in patients with advanced HCC, in which seven of 23 advanced patients achieved PR status, the disease control rate achieved $65.2 \%$, and the median OS achieved 13.8 months (unpublished data). These studies suggested that apatinib is an effective agent in the treatment of advanced HCC. Indeed, these findings surpassed our expectation that some patients with locally advanced HCC patients would have a chance to be "down-staged" and receive radical resection after the treatment of apatinib. And it indeed improved the prognosis of the five patients. For patient 1, as of May 2018, there has been no recurrence and metastasis, and the disease-free survival is 13 months. For patient 5, lung metastasis was found at April 2019. No recurrence was found for the other 3 patients. All patients were alive at the latest follow-up (August 20, 2019).

VEGFR-2 is a major signal transducer for angiogenesis induced by VEGF (32). Apatinib can decrease tumor microvessel density and thus slow, or even stop, tumor growth and development by blocking VEGF signaling. However, the mechanism of apatinib may not be limited to the anti-VEGFR2 effect. A study showed that low dose of apatinib alleviated hypoxia and re-shaped the immunosuppressed tumor microenvironment into a permissive anti-tumor immunity (33). It may contribute to the high response rate. Although the underlying mechanism is still unclear and the relatively high response rate warrants more intensive study.

Apatinib has been approved as a third-line treatment for gastric cancer (34). Recent studies showed that many other cancer types are also sensitive to apatinib, such as ovarian cancer, breast cancer and non-small cell lung cancer (35-37). As apatinib can optimizes tumor microenvironment, it may enhance the therapeutic effect of immunotherapy. A study demonstrated that apatinib potentiates antitumor effect of PD-1/PD-L1 blockade in lung cancer (33). A phase I study showed that the combination of SHR-1210 (anti-
PD-1 antibody) with apatinib achieved an ORR of $50.0 \%$ in patients with advanced HCC (21). These studies showed that the anti-tumor ability was indeed enhanced when apatinib combined with immunotherapy. As many cancers are sensitive to apatinib, so apatinib may be an effective option for combination therapy.

In conclusion, apatinib is an effective drug in the treatment of advanced HCC. Some patients with locally advanced HCC may benefit from "down-staging" and subsequent surgical resection, which could markedly improve the prognosis of this devastating disease.

\section{Acknowledgements}

This article has been edited by Professor James Liu.

Funding: This study was supported by the National Natural Science Foundation of China (81672884), the National Science and Technology Major Project (2017ZX10203207004-005).

\section{Footnote}

Reporting Checklist: The authors have completed the CARE reporting checklist. Available at http://dx.doi.org/10.21037/ tcr-19-3019

Conflicts of Interest: All authors have completed the ICMJE uniform disclosure form (available at http://dx.doi. org/10.21037/tcr-19-3019). The authors have no conflicts of interest to declare.

Ethical Statement: The authors are accountable for all aspects of the work in ensuring that questions related to the accuracy or integrity of any part of the work are appropriately investigated and resolved. The study was conducted in accordance with the Declaration of Helsinki (as revised in 2013). Written informed consent was obtained from the patient for publication of this Case report and any accompanying images.

Open Access Statement: This is an Open Access article distributed in accordance with the Creative Commons Attribution-NonCommercial-NoDerivs 4.0 International License (CC BY-NC-ND 4.0), which permits the noncommercial replication and distribution of the article with the strict proviso that no changes or edits are made and the original work is properly cited (including links to both the formal publication through the relevant DOI and the license). 
See: https://creativecommons.org/licenses/by-nc-nd/4.0/.

\section{References}

1. Liu K, McCaughan GW. Epidemiology and Etiologic Associations of Non-alcoholic Fatty Liver Disease and Associated HCC. Adv Exp Med Biol 2018;1061:3-18.

2. Minagawa $M$, Makuuchi $M$. Treatment of hepatocellular carcinoma accompanied by portal vein tumor thrombus. World J Gastroenterol 2006;12:7561-7.

3. EASL Clinical Practice Guidelines. Management of hepatocellular carcinoma. J Hepatol 2018;69:182-236.

4. Llovet JM, Ricci S, Mazzaferro V, et al. Sorafenib in advanced hepatocellular carcinoma. N Engl J Med 2008;359:378-90.

5. Cheng AL, Kang YK, Chen Z, et al. Efficacy and safety of sorafenib in patients in the Asia-Pacific region with advanced hepatocellular carcinoma: a phase III randomised, double-blind, placebo-controlled trial. Lancet Oncol 2009;10:25-34.

6. Kudo M, Finn RS, Qin S, et al. Lenvatinib versus sorafenib in first-line treatment of patients with unresectable hepatocellular carcinoma: a randomised phase 3 noninferiority trial. Lancet 2018;391:1163-73.

7. Qin S, Bai Y, Lim HY, et al. Randomized, multicenter, open-label study of oxaliplatin plus fluorouracil/leucovorin versus doxorubicin as palliative chemotherapy in patients with advanced hepatocellular carcinoma from Asia. J Clin Oncol 2013;31:3501-8.

8. Vilgrain V, Pereira H, Assenat E, et al. Efficacy and safety of selective internal radiotherapy with yttrium-90resin microspheres compared with sorafenib in locally advanced and inoperable hepatocellular carcinoma (SARAH): an open-label randomised controlled phase 3 trial. Lancet Oncol 2017;18:1624-36.

9. Chow PKH, Gandhi M, Tan SB, et al. SIRveNIB: Selective Internal Radiation Therapyvs.Sorafenib in AsiaPacific Patients With Hepatocellular Carcinoma. J Clin Oncol 2018;36:1913-21.

10. Johnson PJ, Qin S, Park JW, et al. Brivanib vs sorafenib as first-line therapy in patients with unresectable, advanced hepatocellular carcinoma: results from the randomized phase III BRISK-FL study. J Clin Oncol 2013;31:3517-24.

11. Cainap C, Qin S, Huang WT, et al. Linifanib vs Sorafenib in patients with advanced hepatocellular carcinoma: results of a randomized phase III trial. J Clin Oncol 2015;33:172-9.

12. Cheng AL, Kang YK, Lin DY, et al. Sunitinib vs sorafenib in advanced hepatocellular cancer: results of a randomized phase III trial. J Clin Oncol 2013;31:4067-75.

13. Hamaoka M, Kobayashi T, Kuroda S, et al. Hepatectomy after down-staging of hepatocellular carcinoma with portal vein tumor thrombus using chemoradiotherapy: A retrospective cohort study. Int J Surg 2017;44:223-8.

14. Yamamoto Y, Ikoma H, Morimura R, et al. Posthepatectomy survival in advanced hepatocellular carcinoma with portal vein tumor thrombosis. World J Gastroenterol 2015;21:246-53.

15. Xu JF, Liu XY, Wang S, et al. Surgical treatment for hepatocellular carcinoma with portal vein tumor thrombus: a novel classification. World J Surg Oncol 2015;13:86-90.

16. Ye JZ, Zhang YQ, Ye HH, et al. Appropriate treatment strategies improve survival of hepatocellular carcinoma patients with portal vein tumor thrombus. World J Gastroenterol 2014;20:17141-7.

17. Zhang XP, Wang K, Li N, et al. Survival benefit of hepatic resection versus transarterial chemoembolization for hepatocellular carcinoma with portal vein tumor thrombus: a systematic review and meta-analysis. BMC Cancer 2017;17:902-15.

18. Villanueva A. Hepatocellular Carcinoma. N Engl J Med 2019;380:1450-62.

19. Kudo M. Systemic Therapy for Hepatocellular Carcinoma: Latest Advances. Cancers (Basel) 2018;10:412-28.

20. Makker V, Rasco D, Vogelzang NJ, et al. Lenvatinib plus pembrolizumab in patients with advanced endometrial cancer: an interim analysis of a multicentre, open-label, single-arm, phase 2 trial. Lancet Oncol 2019;20:711-8.

21. Xu J, Zhang Y, Jia R, et al. Anti-PD-1 Antibody SHR-1210 Combined with Apatinib for Advanced Hepatocellular Carcinoma, Gastric, or Esophagogastric Junction Cancer: An Open-label, Dose Escalation and Expansion Study. Clin Cancer Res 2019;25:515-23.

22. El Dika I, Khalil DN, Abou-Alfa GK. Immune checkpoint inhibitors for hepatocellular carcinoma. Cancer 2019;125:3312-9.

23. Tomizawa K, Miura Y, Fukui Y, et al. Curative resection for locally advanced sigmoid colon cancer using neoadjuvant chemotherapy with FOLFOX plus panitumumab: A case report. Int J Surg Case Rep 2017;31:128-31.

24. Nagasaki T, Akiyoshi T, Ueno M, et al. Laparoscopic abdominosacral resection for locally advanced primary rectal cancer after treatment with mFOLFOX6 plus bevacizumab, followed by preoperative chemoradiotherapy. Asian J Endosc Surg 2014;7:52-5.

25. Suenaga M, Fujimoto Y, Matsusaka S, et al. Perioperative 
FOLFOX4 plus bevacizumab for initially unresectable advanced colorectal cancer (NAVIGATE-CRC-01). Onco Targets Ther 2015;8:1111-8.

26. Kitajima T, Hatano E, Mitsunori Y, et al. Complete pathological response induced by sorafenib for advanced hepatocellular carcinoma with multiple lung metastases and venous tumor thrombosis allowing for curative resection. Clin J Gastroenterol 2015;8:300-5.

27. Curtit E, Thiery-Vuillemin A, Nguyen T, et al. Complete histologic response induced by sorafenib in advanced hepatocellular carcinoma: a case report. J Clin Oncol 2011;29:e330-2.

28. Irtan S, Chopin-Laly X, Ronot M, et al. Complete regression of locally advanced hepatocellular carcinoma induced by sorafenib allowing curative resection. Liver Int 2011;31:740-3.

29. Kermiche-Rahali S, Di Fiore A, Drieux F, et al. Complete pathological regression of hepatocellular carcinoma with portal vein thrombosis treated with sorafenib. World J Surg Oncol 2013;11:171-5.

30. Kong Y, Sun L, Hou Z, et al. Apatinib is effective for treatment of advanced hepatocellular carcinoma. Oncotarget 2017;8:105596-605.

31. Williet N, Dubreuil O, Boussaha T, et al. Neoadjuvant sorafenib combined with gemcitabine plus oxaliplatin in advanced hepatocellular carcinoma. World J Gastroenterol
2011;17:2255-8.

32. Veeravagu A, Hsu AR, Cai W, et al. Vascular endothelial growth factor and vascular endothelial growth factor receptor inhibitors as anti-angiogenic agents in cancer therapy. Recent Pat Anticancer Drug Discov 2007;2:59-71.

33. Zhao S, Ren S, Jiang T, et al. Low-Dose Apatinib Optimizes Tumor Microenvironment and Potentiates Antitumor Effect of PD-1/PD-L1 Blockade in Lung Cancer. Cancer Immunol Res 2019;7:630-43.

34. Li J, Qin S, Xu J, et al. Randomized, Double-Blind, Placebo-Controlled Phase III Trial of Apatinib in Patients With Chemotherapy-Refractory Advanced or Metastatic Adenocarcinoma of the Stomach or Gastroesophageal Junction. J Clin Oncol 2016;34:1448-54.

35. Miao M, Deng G, Luo S, et al. A phase II study of apatinib in patients with recurrent epithelial ovarian cancer. Gynecol Oncol 2018;148:286-90.

36. Hu X, Zhang J, Xu B, et al. Multicenter phase II study of apatinib, a novel VEGFR inhibitor in heavily pretreated patients with metastatic triple-negative breast cancer. Int J Cancer 2014;135:1961-9.

37. Wu F, Zhang S, Xiong A, et al. A Phase II Clinical Trial of Apatinib in Pretreated Advanced Non-squamous Non-small-cell Lung Cancer. Clin Lung Cancer 2018;19:e831-42.
Cite this article as: Hou Z, Zhu K, Yang X, Zhou H, Chen P, Yu G, Zhu X, Cui Y, Song T, Li Q, Li H, Zhang T. Resection of "down-staged" advanced hepatocellular carcinoma after treatment with the VEGFR2 inhibitor apatinib: five cases report. Transl Cancer Res 2020;9(8):4999-5007. doi: 10.21037/tcr19-3019 